\title{
Activity of Midbrain Reticular Formation and Neocortex during the Progression of Human Non-Rapid Eye Movement Sleep
}

\author{
Naofumi Kajimura, ${ }^{1}$ Makoto Uchiyama, ${ }^{2}$ Yutaka Takayama, ${ }^{1}$ Sunao Uchida, ${ }^{3}$ Takeshi Uema, ${ }^{1}$ Masaaki Kato, ${ }^{1}$ \\ Masanori Sekimoto, ${ }^{1}$ Tsuyoshi Watanabe, ${ }^{1}$ Toru Nakajima, ${ }^{4}$ Satoru Horikoshi, ${ }^{1}$ Kenichi Ogawa, ${ }^{1}$ \\ Masami Nishikawa, ${ }^{2}$ Masahiko Hiroki, ${ }^{5}$ Yoshihisa Kudo, ${ }^{2}$ Hiroshi Matsuda, ${ }^{1}$ Masako Okawa, ${ }^{2}$ and \\ Kiyohisa Takahashi ${ }^{1,2}$ \\ ${ }^{1}$ National Center Hospital for Mental, Nervous, and Muscular Disorders, National Center of Neurology and Psychiatry \\ (NCNP), Kodaira 187-8551, Japan, ${ }^{2}$ National Institute of Mental Health, NCNP, Ichikawa 272-0827, Japan, ${ }^{3}$ Tokyo \\ Institute of Psychiatry, Tokyo 156-0057, Japan, 4Teikyo University School of Medicine, Kawasaki 213-0001, Japan, and \\ 5Tokyo Metropolitan Neurological Hospital, Fuchu 183-0042, Japan
}

To clarify the neural correlates and brain activity during the progression of human non-rapid eye movement (NREM) sleep, we examined the absolute regional cerebral blood flow (rCBF) during light and deep NREM sleep and during wakefulness in normal humans using positron emission tomography with $\mathrm{H}_{2}{ }^{15} \mathrm{O}$. Relative changes in rCBF during light and deep NREM sleep in comparison to the rCBF during wakefulness were also analyzed. During light NREM sleep, the rCBF in the midbrain, in contrast to that in the pons and thalamic nuclei, did not decrease when compared to that during wakefulness, whereas rCBF decreased in the left medial frontal gyrus, left inferior frontal gyrus, and left inferior parietal gyrus of the neocortex. During deep NREM sleep, the $\mathrm{rCBF}$ in the midbrain tegmentum decreased, and there was a marked and bilateral decrease in the $\mathrm{rCBF}$ in all neocortical regions except for the perirolandic areas and the occipital lobe. There have been three groups of brain structures, each representing one type of deactivation during the progression of NREM sleep. The activity of the midbrain reticular formation is maintained during light NREM sleep and therefore represents a key distinguishing characteristic between light and deep NREM sleep. Selective deactivation of heteromodal association cortices, including those related to language, occurs with increasingly deep NREM sleep, which supports the recent theory that sleep is not a global, but it is a local process of the brain.

Key words: NREM sleep; positron emission tomography; cerebral blood flow; midbrain reticular formation; ascending reticular activating system; selective deactivation; heteromodal association cortex
Sleep consists of two different types, rapid eye movement (REM) sleep and non-rapid eye movement (NREM) sleep, which is further classified into stages 1,2,3, and 4, according to the degree of electroencephalogram (EEG) slowing (Rechtschaffen and Kales, 1968). Since its discovery (Aserinsky and Kleitman, 1953), REM sleep has been the principal focus of sleep research, because this sleep is associated with characteristic phenomena, including ocular saccades, muscular atonia, and dreaming (Jones, 1991). However, NREM sleep makes up $\sim 80 \%$ of total sleep (Mendelson, 1987) and is neurophysiologically distinct from waking, whereas waking and REM sleep are neurophysiologically similar (Llinas and Pare, 1991; Steriade et al., 1993b). Animal studies have revealed that centrencephalic structures, including the basal forebrain (Mcginty and Szymusiac, 1989) and the thalamic nuclei (Steriade et al., 1993a), together with decreased activity in the ascending reticular activating system (Moruzzi and Magoun, 1949; Steriade and McCarley, 1990), are associated with

\footnotetext{
Received May 17, 1999; revised Aug. 2, 1999; accepted Sept. 3, 1999.

This work was supported by grants from the Science and Technology Agency, the Ministry of Education, Science, and Culture, and the Ministry of Health and Welfare of Japan. We thank Dr. R. S. J. Frackowiak for his critical comments and suggestions and Dr. T. Okuma for his helpful suggestions.

Correspondence should be addressed to Dr. Naofumi Kajimura, National Center Hospital for Mental, Nervous, and Muscular Disorders, National Center of Neurology and Psychiatry, 4-1-1 Ogawahigashi-cho, Kodaira, Tokyo 187-8551, Japan. E-mail: kajimura@sa2.so-net.ne.jp.

Copyright (C) 1999 Society for Neuroscience 0270-6474/99/1910065-09\$05.00/0
}

NREM sleep. However, the relevance of these findings in animals to humans is unclear. Although some $\mathrm{H}_{2}{ }^{15} \mathrm{O}$ positron emission tomography (PET) studies on human NREM sleep have recently been reported (Braun et al., 1997; Hofle et al., 1997; Maquet et al., 1997), these studies focused only on slow wave sleep (SWS) corresponding to deep NREM sleep, which comprises $<30 \%$ of NREM sleep (Mendelson, 1987), or examined the relationship between regional cerebral blood flow ( $\mathrm{rCBF}$ ) and delta activity or spindle activity during NREM sleep. Therefore, detailed analysis has not yet been done during light NREM sleep. Additionally, absolute rCBF rates, which may be more meaningful than normalized rates, were not reported in these studies. Thus, neural correlates and brain activity during the progression of human NREM sleep, especially those during light sleep, remain elusive. In this study, we examined the absolute rates of rCBF during light and deep NREM sleep and during relaxed wakefulness in normal subjects using high-resolution PET with $\mathrm{H}_{2}{ }^{15} \mathrm{O}$. In addition, relative changes of rCBF during light and deep NREM sleep in comparison to the rCBF during wakefulness were analyzed.

\section{MATERIALS AND METHODS}

Subjects. Eighteen, healthy, right-handed, male university students (age, $21.9 \pm 1.5$ years; range, $19-24$ years) served as the subjects. All gave written informed consent before their participation in the study, which was approved by the Intramural Research Board of the National Center of Neurology and Psychiatry.

Experimental procedure. Each participant visited the sleep laboratory 
$2 \mathrm{~d}$ before the night of the experiment. Each was instructed not to sleep on the night before the experiment and remained under constant observation throughout that time. An ambulatory mini motionlogger actigraph, which measures wrist activity every $30 \mathrm{sec}$ and distinguishes sleep from wakef ulness with a high degree of accuracy (Cole et al., 1992), was placed on the subject's nondominant wrist. On the night of the experiment, the participant came to the laboratory at 7:00 P.M., and previous total sleep deprivation was confirmed by checking the wrist activity data monitored by the actigraph. The experiment was performed only on subjects in whom total sleep deprivation was verified by the actigraph. Electrodes were attached for polysomnography (PSG), and each participant lay on a scanner couch, with the head stabilized by an individually molded thermoplastic face mask secured to the headholder. A venous line was inserted into the right median antebrachial vein for injection of tracer, and an arterial line was inserted into the left radial artery for blood sample measurement of radioactivity throughout the scanning period. EEGs were recorded from disk electrodes placed at Fp1, Fp2, F3, F4, C3, C4, P3, P4, F7, F8, T5, T6, Fz, Cz, and Pz using A1 + A2 for reference. Monopolar electrooculograms were recorded from both canthi, and bipolar electromyograms were recorded from the chin. Sleep stage scoring was performed according to the standardized sleep manual of Rechtschaffen and Kales (1968). Lights were turned out, and PSG recording was started at $\sim$ 10:00 P.M. A maximum of 12 intravenous injections of $\mathrm{H}_{2}{ }^{15} \mathrm{O}$ were administered during NREM sleep, REM sleep, and relaxed wakefulness under continuous PSG monitoring. $\mathrm{H}_{2}{ }^{15} \mathrm{O}$ was injected when the PSG showed the characteristic sleep patterns for light and deep NREM sleep or REM sleep over the 12 min period necessary for $\mathrm{H}_{2}{ }^{15} \mathrm{O}$ production. Ninety second PET scans were collected with a high-resolution PET scanner in three-dimensional mode. Light NREM sleep includes stages 1 and 2 sleep, and deep NREM sleep corresponding to SWS includes stages 3 and 4 sleep, according to the standardized sleep manual of Rechtschaffen and Kales (1968). The subjects were awakened at $\sim 8: 00$ A.M., and scans during wakefulness were taken when $\alpha$ activity was predominant. Final evaluation of sleep stage scoring for each $90 \mathrm{sec}$ period during PET scanning was confirmed later using $\mathrm{C} 3$ recording by visual analysis.

PET procedure. PET scans were obtained using a Siemens ECAT EXACT HR 961 scanner. The camera, having an axial field of view of $150 \mathrm{~mm}$, acquired data simultaneously from 47 consecutive axial planes. An image resolution of $3.8 \times 3.8 \times 4.7 \mathrm{~mm}$ was obtained after backprojection and filtering (Hanning filter, cutoff frequency 0.5 cycles per pixel). The reconstructed image was displayed in a matrix of $128 \times 128 \times$ 47 voxel format (voxel size, $1.732 \times 1.732 \times 3.125 \mathrm{~mm}$ ). A $10 \mathrm{~min}$ transmission scan using a retractable, rotating 68 gallium/68 germanium source with three rods was performed to correct for tissue attenuation and background activity before acquisition of the emission data. For each PET scan, $7 \mathrm{mCi}_{2}{ }^{15} \mathrm{O}$ was automatically flushed intravenously over 15 $\mathrm{sec}$ as a bolus. The total radioactivity administered to each subject was $<1 \mathrm{mSv}$. Scanning was manually commenced $1 \mathrm{sec}$ after the initial rise of head counts, and was continued for $90 \mathrm{sec}$. Arterial blood was sampled automatically throughout the scanning period using a flow-through radioactivity monitor (PICO COUNT; Bioscan, Washington, DC). Absolute $\mathrm{rCBF}$ images were produced using arterial time activity data by the autoradiographic method (Herscovitch et al., 1983; Raichle et al., 1983).

Data analysis. Data were analyzed on a Sun Sparc 20 workstation (Sun Computers Japan, Tokyo, Japan) using Analyze version 7.5.4. image display software (Biodynamic Research Unit, Mayo Foundation, Rochester, MN) and statistical parametric mapping (SPM) software (Friston et al., 1995) (SPM 96; Wellcome Department of Cognitive Neurology, London, UK). Before image analysis, all of the scans of each subject were realigned to the first scan on a voxel-by-voxel basis using the SPM software. The scans were transformed into stereotactic space using both linear and nonlinear three-dimensional transformation methods to allow intersubject averaging. The stereotactically normalized scans contained 68 planes (voxel size, $2 \times 2 \times 2 \mathrm{~mm}$ ) corresponding to the atlas of Talairach and Tournoux (1988). Smoothing with a $12 \mathrm{~mm}$ Gaussian kernel produced a resolution of $17 \times 17 \times 20 \mathrm{~mm}$ for the final image. To evaluate real changes in $\mathrm{rCBF}$ that occurred during the progression of human NREM sleep, the absolute rates of rCBF during light and deep NREM sleep and during wakefulness were analyzed and compared in this study. Global flow normalization method was not used in the absolute $\mathrm{rCBF}$ analysis. Additionally, relative changes of $\mathrm{rCBF}$ were compared using analysis of covariance (ANCOVA) on CBF values to further explore the data (Friston et al., 1991).

After the appropriate design matrix was specified, the subject and condition were estimated according to general linear model at each and every voxel. These analyses generated SPM $\{\mathrm{t}\}$ maps that were subsequently transformed to the unit normal distribution (SPM $\{Z\})$. The exact level of significance of volumes of difference between conditions was characterized by peak amplitude. Clusters of voxels that had a peak $z$ score of $>3.09$ (threshold $p<0.001$ ) were considered to show significant difference. A corrected $p$ value of 0.05 was used as a statistical cluster threshold.

\section{RESULTS}

Thirteen (age, $21.2 \pm 1.9$ years; range, 19-24 years) of 18 subjects were able to sleep sufficiently for PET scanning under the controlled conditions of our study. Not all 13 subjects provided us with complete sets of light and deep NREM sleep and wakefulness. Scanning was performed in 11 subjects during light and deep NREM sleep and wakef ulness, in one subject during light NREM sleep and wakefulness, and in the remaining subject during deep NREM sleep and wakefulness. Thus, the number of subjects included in the pairwise contrasts were as follows: light NREM sleep-wakefulness, 12 subjects; deep NREM sleep-wakefulness, 12 subjects; and light-deep NREM sleep, 11 subjects. Scanning was also performed in eight of these subjects during REM sleep. All of these scans during sleep were acquired after sleep onset, that is, after the polysomnogram showed stage 2 sleep characterized by the appearance of spindles or K-complexes. Scanning for NREM sleep was completed in the early part of the night, whereas that for REM sleep was done in the early morning. A total of 29 scans for light NREM sleep (five scans for stage 1 and 24 scans for stage 2 sleep), 30 scans for deep NREM sleep (13 scans for stage 3 and 17 scans for stage 4 sleep), 14 scans for REM sleep, and 37 scans for wakefulness were successfully obtained. All the scans for stage 1 sleep used in the present study were characterized by vertex sharp waves. The results of rCBF during REM sleep will soon be reported separately.

The absolute rates of global CBF during light and deep NREM sleep and during wakefulness were $33.2 \pm 5.3,31.4 \pm 3.3$, and $35.2 \pm 7.2$, respectively $\left(\mathrm{ml} \cdot 100 \mathrm{gm}^{-1} \cdot \mathrm{min}^{-1}\right.$, mean $\left.\pm \mathrm{SD}\right)$. There was a significant difference in the global CBF during light sleep, deep sleep, and wakefulness, as assessed by ANOVA ( $F=$ $3.685 ; p=0.028$ ). The global CBF during deep sleep was significantly lower than that during wakefulness (Bonferroni test, $p<$ 0.01 ), whereas a significant difference in global CBF was not seen between light sleep and wakefulness or between light and deep sleep.

The absolute rCBF in the pons, cerebellum, thalamus, putamen, anterior cingulate gyrus [Brodmann's area (BA) 24], and left neocortical regions, including the medial frontal gyrus (BA 8, 9, 46), inferior frontal gyrus (BA 44), and the inferior parietal gyrus (BA 39, 40) during light NREM sleep was lower than that in the respective area during wakefulness (Table 1, Fig. 1). In addition, absolute rCBF was reduced during deep NREM sleep in the midbrain tegmentum, cerebellar vermis, basal forebrain, caudate nucleus, and posterior cingulate gyrus as well as in bilateral neocortical regions (except the perirolandic areas and the occipital lobe) in comparison to wakefulness (Table 2, Fig. 2). Thus, the rCBF in the midbrain tegmentum remained unchanged during light sleep but decreased during deep sleep (Figs. 1, 2). No significant increase in absolute $\mathrm{rCBF}$ was observed in any region of the brain during light or deep sleep relative to wakefulness.

The comparison of absolute rCBF between light and deep NREM sleep revealed that the $\mathrm{rCBF}$ in the pons, midbrain tegmentum, cerebellar vermis, caudate nucleus, and the thalamus significantly decreased during deep sleep when compared with 
Table 1. Brain regions in which the absolute rCBF values showed a significant decrease during light NREM sleep compared to that during wakefulness

\begin{tabular}{|c|c|c|c|c|c|c|}
\hline Region & Brodmann's area & $z$ score & $x$ & $y$ & $z$ & corrected $p$ \\
\hline Pons & & 4.91 & 0 & -20 & -38 & $<0.001$ \\
\hline Left cerebellar hemisphere & & 5.15 & -38 & -52 & -48 & $<0.001$ \\
\hline Right cerebellar hemisphere & & 4.44 & 52 & -68 & -40 & 0.027 \\
\hline Left thalamus & & 5.30 & -6 & -24 & 10 & 0.001 \\
\hline Right thalamus & & 5.19 & 12 & -26 & 12 & 0.001 \\
\hline Left putamen & & 4.66 & -22 & -6 & 10 & 0.011 \\
\hline Anterior cingulate gyrus & 24 & 4.63 & 10 & 14 & 36 & 0.013 \\
\hline Left inferior frontal gyrus & 44 & 4.89 & -38 & 44 & 2 & 0.004 \\
\hline \multirow[t]{3}{*}{ Left medial frontal gyrus } & 46 & 4.89 & -44 & 38 & 28 & 0.004 \\
\hline & 9 & 4.50 & -38 & 18 & 40 & 0.021 \\
\hline & 8 & 4.30 & -34 & 32 & 44 & 0.047 \\
\hline \multirow[t]{2}{*}{ Left inferior parietal gyrus } & 40 & 5.38 & -54 & -50 & 50 & $<0.001$ \\
\hline & 39 & 4.34 & -52 & -68 & 36 & 0.040 \\
\hline Left insula & & 4.34 & -38 & 2 & 6 & 0.039 \\
\hline
\end{tabular}

Coordinates are defined in the stereotactic space of Talairach and Tournoux (1988), relative to anterior commissure. $x$ is the lateral distance from midline (positive: right), $y$ is the anteroposterior distance from anterior commissure (positive: anterior), and $z$ is the rostrocaudal distance from the bicommissural plane (positive: rostral). Significant level is employed at a height threshold of $p=0.001$, by reference to unit normal distribution $(z=3.09)$, and at a threshold of corrected $p<0.05$.

$\boldsymbol{\alpha}$

\section{Right lateral}

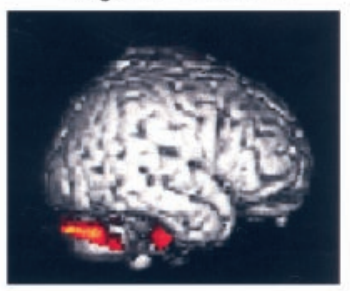

Vertex

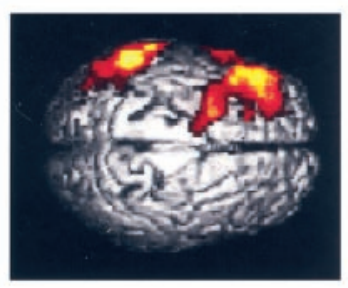

$b$
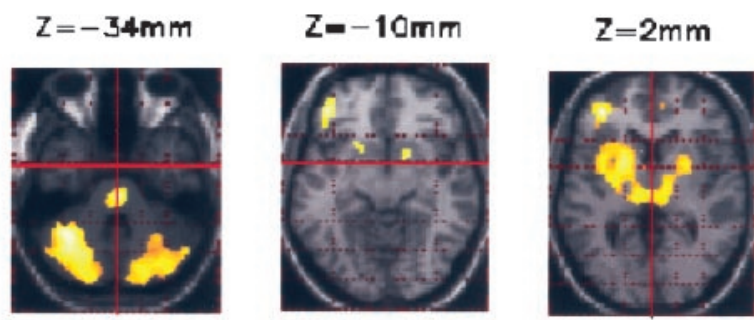

Left lateral
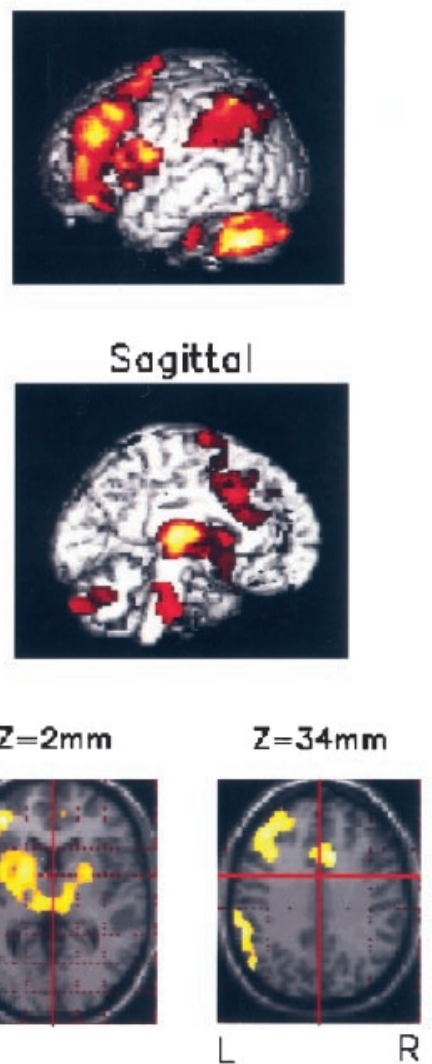

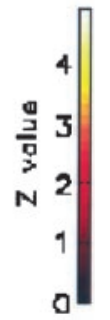

Figure 1. Surface projections $(a)$ and transverse sections $(b)$ of brain areas with significantly decreased absolute rCBF during light NREM sleep when compared with that during wakefulness. An SPM at a height threshold of $p=0.001$, with reference to unit normal distribution $(z=3.09)$, and at an extent threshold of $p=0.05$, is presented. Section numbers $(b)$ refer to the distance from the bicommissural plane. The absolute $\mathrm{rCBF}$ in the pons shows a decrease during light NREM sleep ( $a$, sagittal; $b, z=-34$ ), whereas that in the midbrain remains unchanged ( $a$, sagittal; $b, z=-10$ ). The absolute $\mathrm{rCBF}$ in the left neocortical regions, including the medial frontal and inferior frontal gyri and the inferior parietal gyrus, decreased during light NREM sleep ( $a$, left lateral, vertex; $b, z=2,34$ ). that during light sleep (Table 3, Fig. 3). No significant increase in $\mathrm{rCBF}$ was shown in any region during deep sleep relative to light sleep.

The relative $\mathrm{rCBF}$ in the pons, cerebellum, thalamus, anterior cingulate gyrus (BA 32), and left neocortical regions, including the medial frontal gyrus (BA 10,46) and the inferior parietal gyrus (BA, 40) during light NREM sleep, was lower than that in the respective area during wakefulness (Table 4, Fig. 4). The relative $\mathrm{rCBF}$ was reduced during deep NREM sleep in the pons, midbrain tegmentum, cerebellar vermis, cerebellum, thalamus, putamen, right caudate nucleus, posterior cingulate gyrus (BA, $24)$, and left inferior parietal gyrus (BA, 40) in comparison to relative $\mathrm{rCBF}$ in the same areas during wakefulness (Table 5, Fig. 5). As indicated by the absolute $\mathrm{rCBF}$ analysis, the relative $\mathrm{rCBF}$ in the midbrain tegmentum remained unchanged during light sleep and decreased during deep sleep (Figs. 4, 5). 
Table 2. Brain regions in which the absolute rCBF values showed a significant decrease during deep NREM sleep compared to that during wakefulness

\begin{tabular}{|c|c|c|c|c|c|c|}
\hline Region & Brodmann's area & $z$ score & $x$ & $y$ & $z$ & corrected $p$ \\
\hline Pons & & 7.25 & 0 & -30 & -36 & $<0.001$ \\
\hline Midbrain & & 7.18 & -4 & -26 & -12 & $<0.001$ \\
\hline Left cerebellar hemisphere & & 7.40 & -42 & -68 & -42 & $<0.001$ \\
\hline Right cerebellar hemisphere & & 7.27 & 38 & -76 & -34 & $<0.001$ \\
\hline Cerebellar vermis & & 5.84 & 2 & -60 & -8 & $<0.001$ \\
\hline Left thalamus & & 7.62 & -4 & -22 & 10 & $<0.001$ \\
\hline Right thalamus & & 7.51 & 4 & -22 & 2 & $<0.001$ \\
\hline Left caudate nucleus & & 7.17 & -16 & 4 & 10 & $<0.001$ \\
\hline Right caudate nucleus & & 7.09 & 16 & 6 & 8 & $<0.001$ \\
\hline Left putamen & & 7.04 & -22 & 6 & 4 & $<0.001$ \\
\hline Right putamen & & 6.81 & 16 & 8 & -4 & $<0.001$ \\
\hline Anterior cingulate gyrus & 32 & 5.79 & 4 & 34 & 24 & $<0.001$ \\
\hline Posterior cingulate gyrus & 23 & 6.20 & -2 & -32 & 34 & $<0.001$ \\
\hline Left inferior frontal gyrus & 47 & 5.48 & -20 & 20 & -20 & $<0.001$ \\
\hline Right inferior frontal gyrus & 47 & 6.08 & 18 & 24 & -18 & $<0.001$ \\
\hline Left superior frontal gyrus & 9 & 5.94 & -18 & 58 & 30 & $<0.001$ \\
\hline Right superior frontal gyrus & 11 & 5.62 & 34 & 52 & -14 & $<0.001$ \\
\hline Left medial frontal gyrus & 8 & 5.68 & -48 & 26 & 42 & $<0.001$ \\
\hline Right medial frontal gyrus & 46 & 5.70 & 44 & 44 & 20 & $<0.001$ \\
\hline Left inferior parietal gyrus & 40 & 6.35 & -50 & -64 & 40 & $<0.001$ \\
\hline Right inferior parietal gyrus & 40 & 5.89 & 56 & -60 & 44 & $<0.001$ \\
\hline Left superior parietal gyrus & 7 & 5.34 & -38 & -70 & 52 & $<0.001$ \\
\hline Right superior parietal gyrus & 7 & 4.87 & 38 & -56 & 62 & 0.003 \\
\hline Left medial temporal gyrus & 21 & 4.56 & -66 & -46 & 2 & 0.012 \\
\hline Right medial temporal gyrus & 21 & 4.74 & 66 & -44 & 0 & 0.005 \\
\hline Left inferior temporal gyrus & 20 & 5.66 & -66 & -24 & -16 & $<0.001$ \\
\hline Right inferior temporal gyrus & 37 & 5.07 & 62 & -54 & -10 & 0.001 \\
\hline Left superior temporal gyrus & 38 & 4.74 & -34 & 24 & -24 & 0.005 \\
\hline Right superior temporal gyrus & 42 & 4.61 & 66 & -34 & 22 & 0.009 \\
\hline Left precuneus & 7 & 5.93 & -8 & -70 & 44 & $<0.001$ \\
\hline Right precuneus & 7 & 5.19 & 14 & -82 & 48 & 0.001 \\
\hline Left insula & & 4.67 & -50 & -28 & 20 & 0.007 \\
\hline Right insula & & 5.75 & 40 & 8 & 4 & $<0.001$ \\
\hline
\end{tabular}

Details are the same as in Table 1.

\section{DISCUSSION}

\section{Methodological comments}

Recent functional imaging studies using $\mathrm{H}_{2}{ }^{15} \mathrm{O}$ PET have revealed several interesting findings on brain activity during human NREM and REM sleep. In NREM sleep, marked relative rCBF deactivities were seen in the pons, midbrain, thalamus, and basal forebrain during SWS, which implies that these regions are involved in generating the SWS rhythm (Maquet et al., 1997). It has also been reported that during SWS, heteromodal association areas are selectively deactivated, whereas activity in the primary and secondary sensory areas is preserved (Braun et al., 1997). In addition, negative correlation between EEG delta activity and rCBF has been found most markedly in the thalamus (Hofle et al., 1997). In these previous studies, however, detailed analyses were not done during light NREM sleep, which is a transitional state between wakefulness and SWS and is important in considering sleep-wake mechanisms.

Moreover, the results of these previous studies examined relative changes in $\mathrm{CBF}$, because they examined the correlation between $\mathrm{rCBF}$ and SWS, delta activity, or spindle activity or compared the relative regional distribution of CBF during SWS and wakefulness using ANCOVA. In contrast, we additionally examined the absolute rates of $\mathrm{rCBF}$ during both light and deep NREM sleep and during wakefulness to clarify whether global and extensive changes of CBF during the progression of human NREM sleep might confound interpretation of the results. This dual approach also allowed us to compare our data directly with those already in the literature.

The global CBF during deep NREM sleep was significantly lower than that during wakefulness, whereas a significant difference in global CBF was not seen between light sleep and wakefulness, nor between light and deep sleep. Recently, it is well recognized that a significant decrease in global CBF is observed during SWS (Townsend et al., 1973; Sakai et al., 1980; Madsen et al., 1991; Braun et al., 1997). Therefore the present result on global CBF during deep sleep corresponding to SWS is quite consistent with these results. A previous study also exhibited that CBF declined significantly during light sleep and further declined during deep sleep (Sakai et al., 1980). In the present study, as indicated in this report, there appears to be a decrease in global CBF during light NREM sleep and also to be a continuous decrease in global CBF through wakefulness $(35.2 \pm 7.2 \mathrm{ml} \cdot 100$ 
$\boldsymbol{a}$
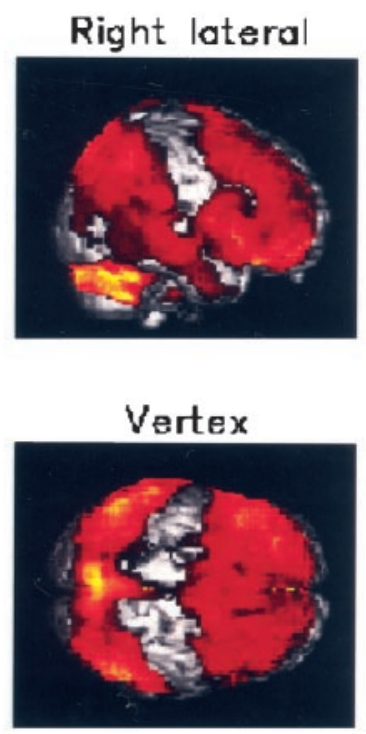

b
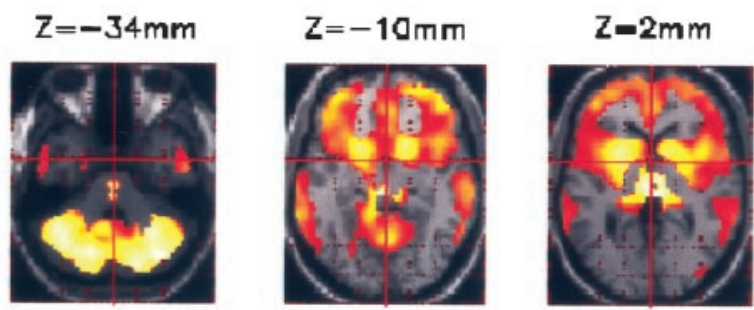

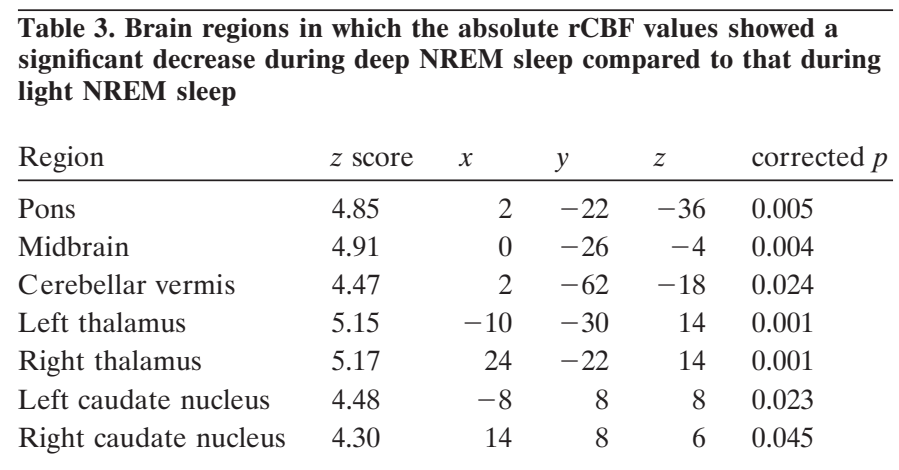

Details are the same as in Table 1.

$\left.\mathrm{gm}^{-1} \cdot \min ^{-1}\right)$, and light $(33.2 \pm 5.3)$ and deep $(31.4 \pm 3.3)$ NREM sleep, although a significant difference was shown only between deep sleep and wakefulness. This possible shift in global $\mathrm{CBF}$ supports the rationale for measuring and comparing absolute rCBF during wakefulness, light, and deep NREM sleep, because relative $\mathrm{rCBF}$ analysis may fail to estimate actual $\mathrm{rCBF}$ changes in this situation.

The relative changes of $\mathrm{rCBF}$ during light NREM sleep in comparison to wakefulness were similar to those found by measuring absolute rCBF. However, relative $\mathrm{rCBF}$ changes during deep NREM sleep in comparison to wakefulness showed some differences from those found by analysis of absolute rCBF. Marked decreases in absolute rCBF in neocortical regions during deep sleep were not detected by relative rCBF analysis. This discrepancy between absolute and relative $\mathrm{rCBF}$ analyses seems to result from the normalization procedure and is attributable to
Figure 2. Surface projections $(a)$ and transverse sections $(b)$ of brain areas with significantly decreased absolute rCBF during deep NREM sleep when compared with that during wakefulness. Details are the same as in Figure 1 . The absolute rCBF in the midbrain tegmentum as well as the pons decreased during deep NREM sleep ( $a$, sagittal; $b, z=-10)$. The absolute rCBF in the bilateral neocortical regions, except the perirolandic areas and the occipital lobe, markedly decreased during deep NREM sleep $(a, b)$.
$\mathbf{L}$
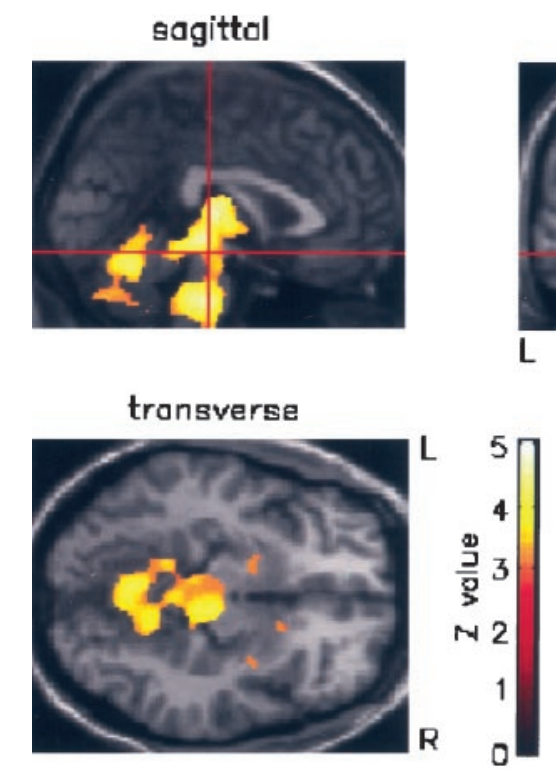

Figure 3. Sagittal $(x=0)$, coronal $(y=-24)$, and transverse $(z=-12)$ sections of brain areas with significantly decreased absolute rCBF during deep NREM sleep when compared with that during light NREM sleep. Details are the same as in Figure 1. The absolute rCBF in the pons, midbrain, cerebellar vermis, caudate nucleus, and the thalamus decreased during deep NREM sleep in comparison to that during light NREM sleep.

prominent decreases in absolute rCBF in the pons, midbrain, thalamus, and the cerebellum during deep NREM sleep compared to wakefulness that are sufficient to influence global flow estimates. Because the global CBF during deep NREM sleep was 
Right lateral
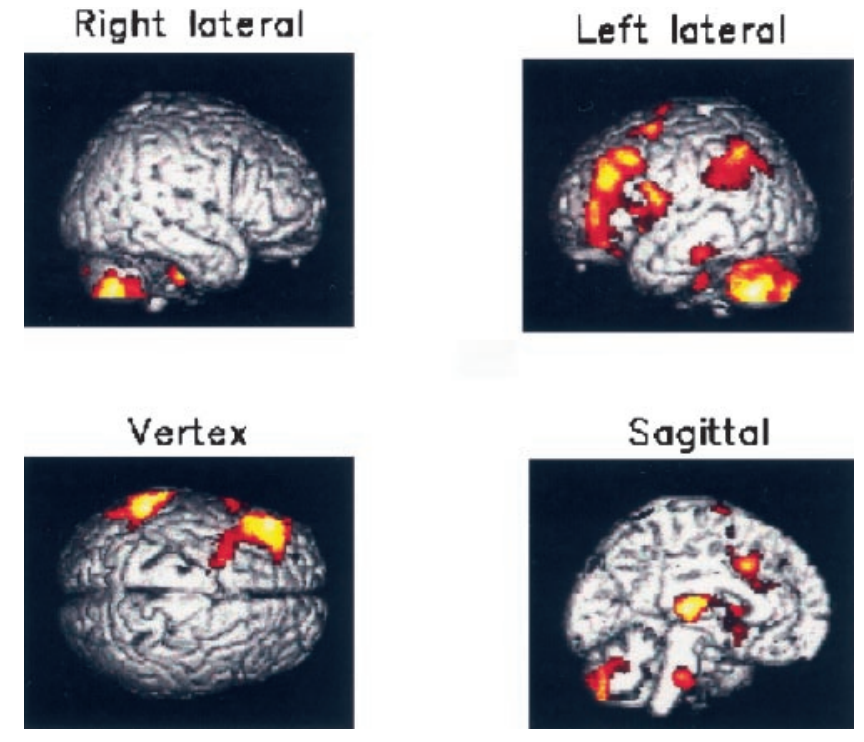

Figure 4. Surface projections of brain areas with significantly decreased relative $\mathrm{rCBF}$ during light NREM sleep when compared with that during wakefulness. An SPM at a height threshold of $p=0.001$, with reference to unit normal distribution $(z=3.09)$, and at an extent threshold of $p=$ 0.05 , is presented. The relative $\mathrm{rCBF}$ in the pons shows a decrease during light NREM sleep, whereas that in the midbrain remains unchanged (sagittal). The relative rCBF in the left neocortical regions, including the medial frontal gyrus and the inferior parietal gyrus, decreased during light NREM sleep (left lateral, vertex).

significantly lower than that during wakefulness, a greater decrease in absolute $\mathrm{rCBF}$ in these centrencephalic structures than in other brain areas attenuated the actual decrease of $\mathrm{rCBF}$ in neocortical regions during deep sleep compared to wakefulness when relative $\mathrm{rCBF}$ changes were analyzed. Therefore, our experimental approach has allowed us to obtain important additional data about the physiology of neocortical regions other than the perirolandic areas and the occipital lobe in deep sleep than could be found by analysis of relative changes of $\mathrm{rCBF}$ alone. Otherwise our data during deep NREM sleep are consistent with the results of previous PET studies that analyzed relative $\mathrm{rCBF}$ changes during SWS (Braun et al., 1997; Maquet et al., 1997).

\section{Activity of the midbrain reticular formation and other centrencephalic structures}

We unexpectedly found that the rCBF in the midbrain tegmentum, in contrast to that in the pons, showed no significant decrease during light sleep, which suggests that the activity of the midbrain reticular formation (MRF) is maintained during this stage of sleep. This is surprising, because it has been believed that the MRF plays a crucial role in the maintenance of wakefulness (Lindsley et al., 1950). On the other hand, a recent PET study showed that the MRF is activated when human subjects went from a relaxed awake state to an attention-demanding reactiontime task (Kinomura et al., 1996). Therefore there may be a possibility that the MRF plays a role, not in the maintenance of wakefulness, but rather in attention or in a temporary elevation of vigilance. Previous reports found that neurotoxic MRF lesions in which axonal pathways were not destroyed did not produce any significant alteration of waking in cats, and that the firing rate of MRF neurons was higher during waking with movement than during quiet waking in rats (Steriade et al., 1982; Denoyer et al., 1991). Furthermore, it is reported that cholinergic neurons ascending from the brainstem, the majority of which originate from the MRF (Mesulam et al., 1983), exhibited strong habituation of phasic sensory responses by repetition of stimulation (Kayama and Ogawa, 1987). These results support our consideration that the MRF may play a primary role in attention or in a temporary elevation of vigilance rather than in the maintenance of wakefulness. Cholinergic neurons in the MRF project directly to the thalamus (dorsal branch) and indirectly to the neocortex via a relay with cholinergic neurons in the basal forebrain (ventral branch) (Woolf and Butcher, 1986). Thus, the activity of the MRF may be maintained during light sleep and may be useful in arousal from this stage of sleep possibly through its ventral cholinergic input to the basal forebrain. On the other hand, it is difficult for humans to awaken from deep sleep, because at that time the activity of the MRF is markedly suppressed together with that of the pons, thalamic nuclei, basal forebrain, and wide neocortical regions.

A sleep center that was specifically activated during light or deep sleep was not detected in this study. However, we suggest that decreased activity in the pons and the thalamic nuclei, which comprise the ascending reticular activating system, is involved in the transition from wakefulness to light sleep in humans. The noradrenergic neurons of the locus coeruleus give rise to widespread projections to the cortex and the thalamus (Lindvall et al., 1974) and act as a tonic activating system in contrast to the cholinergic neurons ascending from the MRF (Kayama and Ogawa, 1987). The serotonergic neurons of the dorsal raphe nuclei project directly to both the thalamus and the neocortex and are also tonically active during wakefulness (Anden et al., 1966; Jacobs and Azmitia, 1992). The noradrenergic, serotonergic, and cholinergic neurons are considered to be related to human waking systems. However, the noradrenergic and serotonergic projections from the brainstem, rather than the cholinergic projection from the MRF, may play a cardinal role in the maintenance of wakefulness, and decreased monoaminergic activity may be partly involved in the occurrence of sleep in humans.

The comparison of absolute rCBF between light and deep NREM sleep revealed a significant decrease in the pons, midbrain tegmentum, cerebellar vermis, caudate nucleus, and the thalamus during deep sleep when compared with light sleep. The pons, midbrain tegmentum, and the thalamus comprise the ascending reticular activating system, and the basal ganglia, including the caudate nucleus, are also associated with cortical activation by the ascending reticular activating system (Moruzzi and Magoun, 1949; Steriade and McCarley, 1990). Therefore, the extent to which activity of the ascending reticular activating system is depressed may determine the transition from light to deep sleep. Previous PET study, which examined the relationship between EEG frequency band activity and normalized rCBF during relaxed wake and stage 1-4 NREM sleep, indicated that negative correlation between delta activity and $\mathrm{rCBF}$ was found most markedly in the thalamus and also in the brainstem reticular formation, cerebellum, anterior cingulate, and orbitofrontal cortex (Hofle et al., 1997). The present finding is supported by the result of this report. Furthermore, it is confirmed by the result of our relative rCBF analysis between deep NREM sleep and wakefulness in which a prominent deactivation in the centrencephalic structures including the pons, midbrain, thalamus, putamen, and the caudate nucleus was found during deep sleep. Thus, the activity level of the ascending reticular activating system, especially that of the MRF, seems important in distinguishing light and deep NREM sleep. 
Table 4. Brain regions in which the relative $\mathrm{rCBF}$ values showed a significant decrease during light NREM sleep compared to that during wakefulness

\begin{tabular}{|c|c|c|c|c|c|c|}
\hline Region & Brodmann's area & $z$ score & $x$ & $y$ & $z$ & corrected $p$ \\
\hline Pons & & 4.75 & 4 & -22 & -38 & 0.028 \\
\hline Left cerebellar hemisphere & & 5.18 & -36 & -62 & -36 & 0.004 \\
\hline Right cerebellar hemisphere & & 5.25 & 42 & -72 & -46 & 0.003 \\
\hline Left thalamus & & 5.44 & -6 & -24 & 10 & 0.001 \\
\hline Right thalamus & & 5.41 & 12 & -24 & 12 & 0.001 \\
\hline Anterior cingulate gyrus & 32 & 4.66 & -2 & 20 & 38 & 0.040 \\
\hline \multirow{2}{*}{ Left medial frontal gyrus } & 10 & 5.65 & -36 & 46 & 4 & $<0.001$ \\
\hline & 46 & 5.65 & -44 & 36 & 28 & $<0.001$ \\
\hline Left inferior parietal gyrus & 40 & 5.87 & -56 & -48 & 50 & $<0.001$ \\
\hline
\end{tabular}

Significant level is employed at a height threshold of $p=0.001$, by reference to unit normal distribution $(z=3.09)$, and at a threshold of corrected $p<0.05$. Details are the same as in Table 1 .

Table 5. Brain regions in which the relative $\mathrm{rCBF}$ values showed a significant decrease during deep NREM sleep compared to that during wakefulness

\begin{tabular}{|c|c|c|c|c|c|c|}
\hline Region & Brodmann's area & $z$ score & $x$ & $y$ & $z$ & corrected $p$ \\
\hline Pons & & 7.32 & 0 & -28 & -38 & $<0.001$ \\
\hline Midbrain & & 6.52 & -4 & -26 & -10 & $<0.001$ \\
\hline Cerebellar vermis & & 5.70 & 18 & -70 & -24 & $<0.001$ \\
\hline Left cerebellar hemisphere & & 7.23 & -42 & -66 & -40 & $<0.001$ \\
\hline Right cerebellar hemisphere & & 6.91 & 18 & -86 & -40 & $<0.001$ \\
\hline Left putamen & & 6.73 & -18 & 4 & 6 & $<0.001$ \\
\hline Right putamen & & 6.29 & 16 & 6 & 8 & $<0.001$ \\
\hline Left thalamus & & 7.67 & -4 & -20 & 8 & $<0.001$ \\
\hline Right thalamus & & 7.55 & 4 & -22 & 2 & $<0.001$ \\
\hline Posterior cingulate gyrus & 24 & 5.14 & 0 & -18 & 38 & 0.005 \\
\hline Left inferior parietal gyrus & 40 & 5.50 & -54 & -48 & 52 & $<0.001$ \\
\hline
\end{tabular}

Details are the same as in Table 1.

\section{Activity of the neocortical regions}

We found that the absolute $\mathrm{rCBF}$ in the left neocortical regions, including the medial and inferior frontal gyri and the inferior parietal gyrus (supramarginal gyrus and angular gyrus), selectively decreased during light sleep. During wakefulness, the frontal cortex performs higher-order processing of sensory information, integrating cognitive information and organizing behavioral responses; the parietal cortex is also associated with higher-order cortical activities, including spatial perception, attention, and language (Mesulam, 1987). Additionally, the left inferior frontal gyrus contains Broca's area, and the left supramarginal and angular gyri are included in Wernicke's area in a broad sense. Therefore NREM sleep is characterized by deactivation of the high-order association cortices, and selective deactivation of language areas may take place during the early stage of NREM sleep. Primary motor and somatosensory, and the visual cortices, which serve only as obligatory relays for the transfer of information to other regions of the brain, may remain functional throughout NREM sleep. These results suggest that frontoparietal higher-order association cortices, especially those for language, need the early and extended recuperative benefits of NREM sleep. It is reported that, during wakefulness, relatively high glucose metabolism is observed in left neocortical regions in right-handed normal subjects even with sensory deprivation (Mazziotta et al., 1982). Therefore the present findings may partly support the recently proposed theory that sleep is not an exclusively global process but rather a local, use-dependent pro-
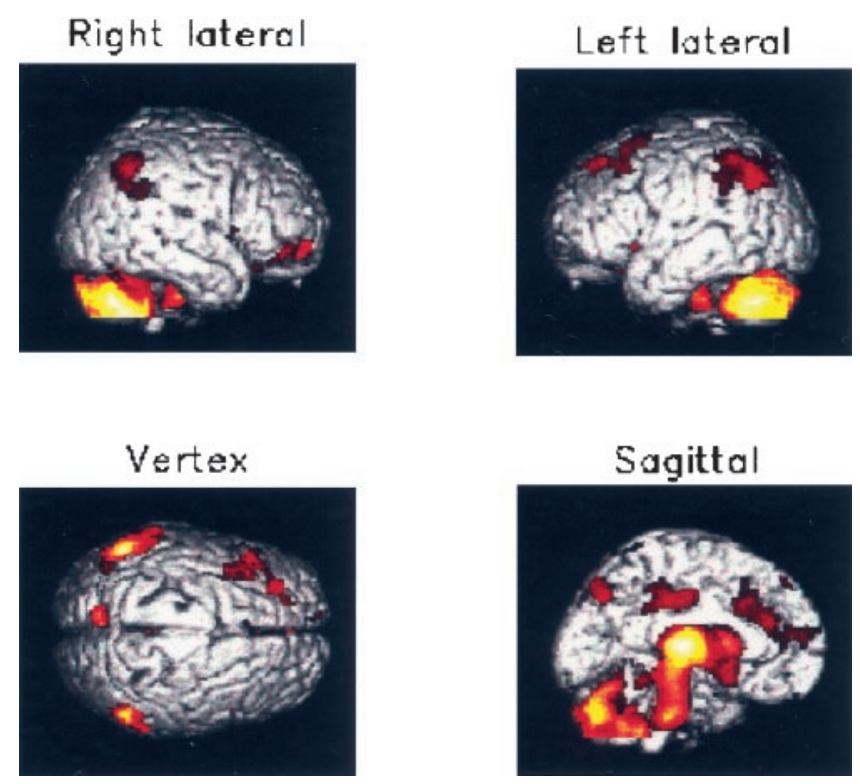

Figure 5. Surface projections of brain areas with significantly decreased relative rCBF during deep NREM sleep when compared with that during wakefulness. Details are the same as in Figure 4. The relative rCBF in the cerebellum and the centrencephalic structures, including the pons, midbrain, and the thalamus shows a marked decreases during deep NREM sleep (Sagittal). 

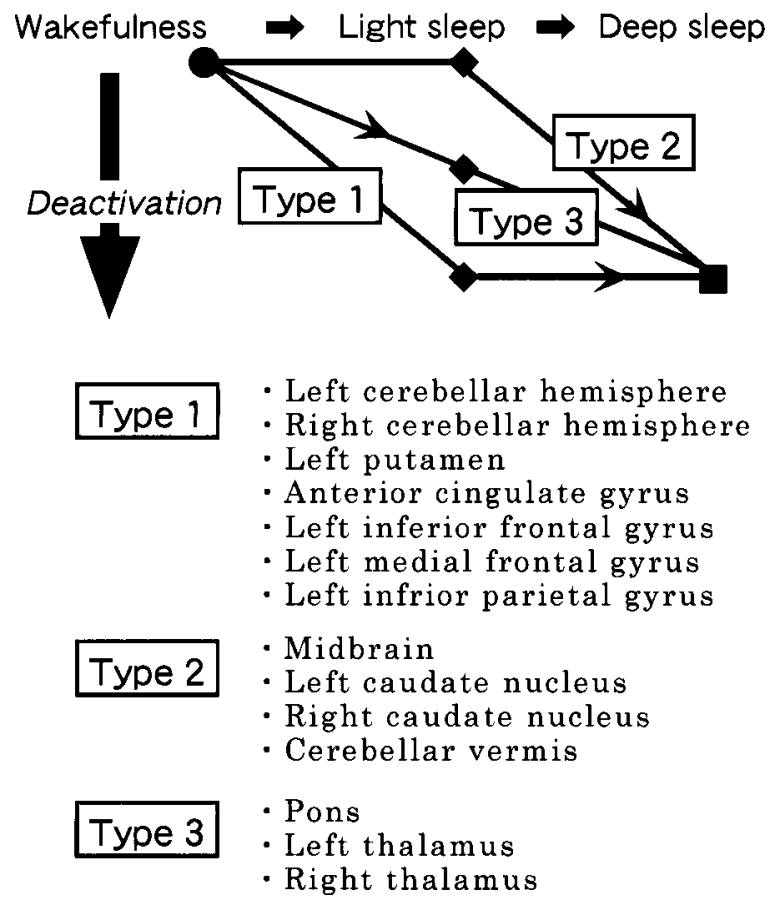

Figure 6. A schematic illustration of three groups of brain structures, each representing one type of deactivation during the progression of NREM sleep. Type 1 group shows deactivation throughout light and deep NREM sleep but represents no difference in deactivation between light and deep sleep, type 2 group shows deactivation finally during deep sleep and exhibits a difference in deactivation between light and deep sleep, and type 3 group shows deactivation progressively through light and deep sleep and represents a difference in deactivation between light and deep sleep.

cess of the brain (Kruger and Obal, 1993; Benington and Heller, 1995), although further study is necessary to clearly address this issue.

\section{Conclusions}

The results of the present study are summarized in Figure 6. There have been three groups of brain structures, each representing one type of deactivation during the progression of human NREM sleep, although the perirolandic areas and the occipital lobe exhibited no deactivation during light and deep NREM sleep. We suggest that type 1 group, including the cerebellar hemisphere, anterior cingulate gyrus, and left frontal and parietal cortical regions shows deactivation during light sleep and also keeps deactivated during deep sleep; type 2 group, including the midbrain, cerebellar vermis, and caudate nucleus keeps activated during light sleep and shows deactivation finally during deep sleep; and type 3 group, including the pons and the thalamus, shows deactivation progressively through light and deep sleep.

We found some particular and significant characteristics of human light NREM sleep that differ from those of deep NREM sleep. The activity of the MRF is maintained during light sleep, possibly to allow humans to wake up quickly in response to various stimuli. The MRF may therefore play a key role in distinguishing light and deep NREM sleep. Higher-order association cortices, especially those related to language, seem to require early and extended rest during NREM sleep, because these areas are considered to be more active during wakefulness.

\section{REFERENCES}

Anden NE, Dahlstrom A, Fuxe K, Larsson K, Olson L, Ungerstedt U (1966) Ascending monoamine neurons to the telencephalon and diencephalon. Acta Physiol Scand 67:313-326.

Aserinsky E, Kleitman N (1953) Regularly occurring periods of eye motility and concomitant phenomena during sleep. Science 118:273-274

Benington JH, Heller HC (1995) Restoration of brain energy metabolism as the function of sleep. Prog Neurobiol 45:347-360.

Braun AR, Balkin TJ, Wesensten NJ, Carson RE, Varga M, Baldwin P, Selbie S, Belenky G, Herscovitch P (1997) Regional cerebral blood flow throughout the sleep-wake cycle: an $\mathrm{H}_{2}{ }^{15} \mathrm{O}$ PET study. Brain 120:1173-1197.

Cole RJ, Kripke DF, Gruen W, Mullaney DJ, Gillin JC (1992) Automatic sleep/wake identification from wrist activity. Sleep 15:461-469.

Denoyer M, Sallanon M, Buda C, Kitahama K, Jouvet M (1991) Neurotoxic lesion of the mesencephalic reticular formation and/or the posterior hypothalamus does not alter waking in the cat. Brain Res 539:287-303.

Friston KJ, Frith CD, Liddle PF, Frackowiak RSJ (1991) Comparing functional (PET) images: the assessment of significant changes. J Cereb Blood Flow Metab 11:690-699.

Friston KJ, Holmes AP, Worsley KJ, Poline JB, Frith CD, Frackowiak RSJ (1995) Statistical parametric maps in functional imaging: a general linear approach. Hum Brain Mapp 2:189-210.

Herscovitch P, Markham J, Raichle ME (1983) Brain blood flow measured with intravenous $\mathrm{H}_{2}{ }^{15} \mathrm{O}$. I. Theory and error analysis. J Nucl Med 24:782-789.

Hofle N, Paus T, Reutens D, Fiset P, Gotman J, Evans AC, Jones BE (1997) Regional cerebral blood flow changes as a function of delta and spindle activity during slow wave sleep in humans. J Neurosci 17:4800-4808.

Jacobs BL, Azmitia EC (1992) Structure and function of the brain serotonin system. Physiol Rev 72:165-229.

Jones BE (1991) Paradoxical sleep and its chemical/structural substrates in the brain. Neuroscience 40:637-656.

Kayama Y, Ogawa T (1987) Electrophysiology of ascending, possibly cholinergic neurons in the rat laterodorsal tegmental nucleus: comparison with monoamine neurons. Neurosci Lett 77:277-282.

Kinomura S, Larsson J, Gulyas B, Roland PE (1996) Activation by attention of the human reticular formation and thalamic intralaminar nuclei. Science 271:512-515.

Kruger JM, Obal Jr F (1993) A neuronal group theory of sleep function. J Sleep Res 2:63-69.

Lindsley DB, Schreiner LH, Knowles WB, Magoun HW (1950) Behavioral and EEG changes following chronic brainstem lesions in the cat. Electroencephalogr Clin Neurophysiol 2:483-498.

Lindvall O, Bjorklund A, Nobin A, Stenevi U (1974) The adrenergic innervation of the rat thalamus as revealed by the glyoxylic acid fluorescence method. J Comp Neurol 154:317-348.

Llinas RR, Pare D (1991) Of dreaming and wakefulness. Neuroscience 44:521-535.

Madsen PL, Schmidt JF, Wildschiodtz G, Friberg L, Hoim S, Vorstrup S, Lassen NL (1991) Cerebral oxygen metabolism and cerebral blood flow in humans during deep and rapid-eye- movement sleep. J Appl Physiol 70:2597-2601.

Maquet P, Degueldre C, Delfiore G, Aerts J, Peters J, Luxen A, Franck G (1997) Functional neuroanatomy of human slow wave sleep. J Neurosci 17:2807-2812.

Mazziotta JC, Phelps ME, Carson RE, Kuhl DE (1982) Tomographic mapping of human cerebral metabolism: sensory deprivation. Ann Neurol 12:435-444.

Mcginty D, Szymusiac R (1989) The basal forebrain and slow wave sleep: mechanistic and functional aspects. In: Slow wave sleep (Wauquiar A, Dugovic C, Radulovacki H, eds). pp 61-73. New York: Raven.

Mendelson WB (1987) Human sleep: research and clinical care. New York: Plenum.

Mesulam MM (1987) Patterns in behavioral neuroanatomy: association areas, the limbic system, and hemispheric specialization. In: Principles of behavioral neurology (Mesulam MM, ed). pp 1-70. Philadelphia: F.A. Davis.

Mesulam MM, Mufson EJ, Wainer BH, Levey AI (1983) Central cholin- 
ergic pathways in the rat: an overview based on an alternative nomenclature (Ch 1-6). Neuroscience 10:1185-1201.

Moruzzi G, Magoun HW (1949) Brainstem reticular formation and activation of the EEG. Electroencephalogr Clin Neurophysiol 1:455-473.

Raichle ME, Martin WRW, Herscovitch P, Mintum MA, Makkam J (1983) Brain blood flow measured with intravenous $\mathrm{H}_{2}{ }^{15} \mathrm{O}$. II. Implementation and validation. J Nucl Med 24:790-798.

Rechtschaffen A, Kales A (1968) A Manual of standardized terminology, techniques and scoring system for sleep stages of human subjects. Bethesda, MD: US Department of Health, Education, and Welfare.

Sakai F, Meyer JS, Karacan I, Derman S, Yamamoto M (1980) Normal human sleep: regional cerebral hemodynamics. Ann Neurol 7:471-478.

Steriade M, McCarley R (1990) Brainstem control of wakefulness and sleep. New York: Plenum.

Steriade M, Oakson G, Ropert N (1982) Firing rates and patterns of midbrain reticular neurons during steady and transitional states of the sleep-waking cycle. Exp Brain Res 46:37-51.

Steriade M, Contreras D, Curro Dossi R, Nunez A (1993a) The slow $(<1 \mathrm{~Hz})$ oscillation in reticular thalamic and thalamocortical neurons: scenario of sleep rhythm generation in interacting thalamic and neocortical networks. J Neurosci 13:3284-3299.

Steriade M, McCormic DA, Sejnowski TJ (1993b) Thalamocortical oscillations in the sleeping and arousal brain. Science 262:679-685.

Talairach J, Tournoux P (1988) Co-planar stereotaxic atlas of the human brain. Stuttgart: Thieme-Verlag.

Townsend RE, Prinz PN, Obrist WD (1973) Human cerebral blood flow during sleep and waking. J Appl Physiol 35:620-625.

Woolf NJ, Butcher LL (1986) Cholinergic systems in the rat brain: 111. projections from the pontomesencephalic tegmentum to the thalamus, tectum, basal ganglia, and basal forebrain. Brain Res Bull 16:603-637. 